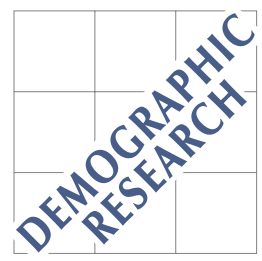

Demographic Research a free, expedited, online journal of peer-reviewed research and commentary in the population sciences published by the Max Planck Institute for Demographic Research Konrad-Zuse Str. 1, D-18057 Rostock · GERMANY www.demographic-research.org

DEMOGRAPHIC RESEARCH

VOLUME 21, ARTICLE 25, PAGES 759-764 PUBLISHED 18 NOVEMBER 2009

http://www.demographic-research.org/Volumes/Vol21/25/

DOI: 10.4054/DemRes.2009.21.25

Formal Relationships 5

\title{
The metastable birth trajectory
}

\section{Robert Schoen}

This article is part of the Special Collection "Formal Relationships". Guest Editors are Joshua R. Goldstein and James W. Vaupel.

(c) 2009 Robert Schoen.

This open-access work is published under the terms of the Creative Commons Attribution NonCommercial License 2.0 Germany, which permits use, reproduction \& distribution in any medium for non-commercial purposes, provided the original author(s) and source are given credit. See http://creativecommons.org/licenses/by-nc/2.0/de/ 


\section{Table of Contents}

$\begin{array}{lll}1 & \text { Introduction } & 759\end{array}$

2 The stable model $\quad 760$

$\begin{array}{lll}3 & \text { The metastable model } & 760\end{array}$

$4 \quad$ History and applications $\quad 762$

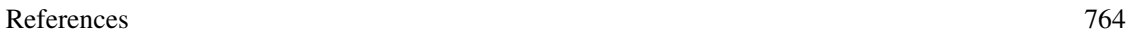




\title{
The metastable birth trajectory
}

\author{
Robert Schoen $^{1}$
}

\begin{abstract}
The metastable model generalizes the stable population model by allowing net maternity to change exponentially over age and time. As a result, the metastable model generates an exponentially quadratic birth trajectory, which is characterized by a constant proportion of births by age of mother. The metastable model is well suited to analyzing steady fertility declines and transitions between two regimes of fixed vital rates.
\end{abstract}

\section{Introduction}

Demography is built on its models. The basic life table, dating from 1662, followed a single birth cohort to the death of its last member. The life table was later conceptualized as a stationary (zero growth) population, a view expanded by the stable population which captures the implications of any given set of age-specific birth and death rates. Recently, the stable population has been extended to the metastable population, which allows the vital rates to change over time. In the stable population, constant vital rates yield (linear) exponential growth in the number of births, while in the metastable model net maternity that increases exponentially over age and time produces quadratic exponential growth in births. This note describes the metastable model, specifies its underlying vital rates and their pattern of change, and provides the model's birth trajectory over time.

\footnotetext{
${ }^{1}$ Pennsylvania State University. E-mail: schoen@pop.psu.edu
} 


\section{The stable model}

Lotka's stable population was the foundational model of $20^{\text {th }}$ century mathematical demography. One elegant equation, generally known as the model's renewal or characteristic equation, captures its essential features. That equation can be written

$$
1=\int e^{-r x} p(x) f(x) d x
$$

where the integration spans all ages $x, p(x)$ is the probability of surviving from birth to exact age $x$ under the specified regime of age-specific mortality, $f(x)$ is the force of fertility at exact age $x$ under the specified regime of age-specific fertility, and $r$, a constant, is Lotka's intrinsic growth rate (Lotka 1998; Preston, Heuveline, and Guillot 2001; Schoen 1988). The stable model is typically applied to a female only population. The stable population Net Reproduction Rate $\left(R_{0}\right)$ is then given by

$$
R_{0}=\int p(x) f(x) d x
$$

where the integral ranges over all reproductive ages and $p(x) f(x)$ is the net maternity function.

Equation (1) can be interpreted in a manner that emphasizes its renewal or regenerative nature. Let us scale the stable population so that there is one birth in our reference year. Then that unit birth arises from the sum, over all reproductive ages, of the $e^{-r x}$ births that occurred $x$ years ago who survived to age $x$ under the specified mortality regime and then gave birth in the reference year according to the specified fertility regime. The renewal equation thus shows how an exponential birth trajectory is perpetuated by constant agespecific fertility and mortality. The realization that constant vital rates yield a population that grows exponentially but maintains a constant age composition is probably the most profound insight in mathematical demography.

\section{The metastable model}

The stable population shows the implications of a given set of age-specific fertility and mortality rates, but is essentially a static model. The metastable model is dynamic because it accommodates monotonic exponential change in vital rates over age and time.

Let the metastable net maternity function be written as

$$
\varphi(x, t)=p(x, t) f(x, t)
$$

where $\varphi(x, t)$ is the net maternity of women age $x$ at time $t, p(x, t)$ is the probability that women born at time $t-x$ will survive to attain age $x$ at time $t$, and $f(x, t)$ is the force of 
fertility for women age $x$ at time $t$. Over time, metastable net fertility changes according to

$$
\varphi(x, t)=\varphi_{0}(x) e^{r x} e^{h t x}
$$

where $\varphi_{0}(x)$ describes a time invariant net maternity function whose $R_{0}=1, r$ is the intrinsic growth rate implied by $\varphi(x, 0)$, and $h$ is a new metastable parameter that specifies how net maternity changes over both age and time.

Equation (4) describes a realistic pattern of change that has been observed empirically and was incorporated into the Coale-Trussell Model Fertility Schedules (Coale and Trussell 1974). When $h>0$, net maternity increases exponentially over time, rising more rapidly at higher ages than at lower ages. That is quite reasonable as higher net maternity typically involves more higher order births, and such births occur mostly at older ages. When $h<0$ and net maternity declines exponentially over time, greater relative declines occur at the older ages as the number of higher order births falls more rapidly. Because net maternity changes exponentially according to $h$, it can quickly depart from demographically reasonable values. In empirical work, $h$ typically has a magnitude no greater than 0.001 . In equation (4), the metastable model is expressed in terms of the net maternity function, with no explicit assumptions made with regard to age-specific fertility and mortality. In contemporary populations, mortality is quite small below the highest age at reproduction, and mortality above the highest age at reproduction does not affect the birth trajectory.

The renewal equation of any closed population can be written

$$
g(t)=\int g(t-x) \varphi(x, t) d x
$$

where $g(t)$ represents the number of births at time $t$ and the integral spans all ages. Let the "trial" metastable birth trajectory be given by

$$
g(t)=g(0) \exp \left[(r+s) t+\frac{h t^{2}}{2}\right]
$$

where the birth trajectory is a quadratic exponential augmented by two linear exponential terms. Inserting equation (6) into equation (5) and using equation (4) yields the relationship

$$
1=\int \exp \left[-s x+\frac{h x^{2}}{2}\right] \varphi_{0}(x) d x
$$

with the integral again ranging over all reproductive ages. In equation (7), all of the time varying functions have dropped out, leaving a Lotka-like equation that serves to 
specify $s$, the second metastable parameter. Parameter $s$ determines the model's constant distribution of births by age of mother. Substantively, $s$ reflects the additional growth in births produced by the interaction between the changing rates of net maternity and the changing age composition of the metastable population.

Equation (6) shows that the metastable birth trajectory grows (positively or negatively) according to a function dominated by a quadratic exponential, where $h$ is the quadratic growth parameter. Supplementary terms add linear exponential growth according to rates $r$ and $s$.

The metastable population accommodates any type of monotonic net maternity change consistent with equation (4), and $h$ can generally be chosen by the analyst. The stable population is the special case of a metastable population with $h=0$. (From equation (6), $s=0$ when $h=0$. In general, $s$ is positive, negative, or zero as $h$ is positive, negative, or zero.) Values for $h, r$, and $\varphi_{0}$ suffice to specify a metastable population. As is the case with the stable population, the metastable model is fully described by explicit, closed form equations. It can be viewed as being in dynamic equilibrium, since the metastable functional relationships and fixed distribution of births by age of mother are preserved as the size, composition, and vital rates of the population change over time in a regular and systematic manner.

\section{History and applications}

A number of demographers have sought to construct models that related changing vital rates to the resultant birth trajectory. In a pioneering work, Coale (1972) approached that question in several ways. In quasi-stable models (previously developed with Paul Demeny), the implications of constant fertility and declining mortality were examined. Coale further explored models with fertility that increased exponentially over time, uniformly at all ages. He found that a quadratic exponential birth sequence resulted, but could not express that trajectory in closed form. Feichtinger and Vogelsang (1978) and Vogelsang and Feichtinger (1979) pursued the matter further, but a closed form solution eluded them as well.

At Johns Hopkins University in the 1990s, Young Kim and I were exploring the implications of patterned changes in rates, largely using discrete models, i.e. Leslie matrices. Zenas Sykes, then retired, had worked closely with Young Kim in the past, and had found a very nice relationship we called the "Sykes transform." That enabled any Leslie matrix to be written as the product of (i) its dominant eigenvalue, (ii) a diagonal matrix containing its dominant right eigenvector, (iii) a base row stochastic matrix (whose elements in all rows sum to 1), and (iv) the inverse of the diagonal eigenvector matrix in (ii) (Sykes 1973). One morning I was fiddling with some Leslie matrices trying to write 
their product analytically. I happened to try expressing the Leslie matrix that projected a population from time $t-1$ to time $t$, denoted $\mathbf{A}_{t}$, by $\mathbf{K}^{t} \mathbf{F} \mathbf{K}^{-t}$, where $\mathbf{F}$ was a row stochastic Leslie matrix and $\mathbf{K}$ was a diagonal matrix, like the Sykes matrix in step (ii) above. The product of those $\mathbf{A}_{t}$ matrices from time 1 through time $t$ simplified, yielding the product $\mathbf{K}^{t}\left(\mathbf{F K}^{-1}\right)^{t} \mathbf{K}^{-1}$. That allowed the long term consequences of rates changing in that particular way to be expressed in a concise, closed form expression. From that result flowed the metastable model and the "trial" birth trajectory used in the previous section. It also revealed the objective of Coale's search. If fertility rates increase exponentially over both age and time, rather than over time alone, a closed form solution for the birth trajectory arises. We presented those results in several papers, including Schoen and Kim (1994) and Kim and Schoen (1996).

Some years later, when I was at Penn State University, I read several excellent papers on gradual declines to stationarity by Li and Tuljapurkar $(1999 ; 2000)$ and by Goldstein (2002). It struck me that the metastable model could also be applied to the analysis of momentum under gradual declines to zero growth. Working with my student, Stefan Jonsson, I developed that idea in Schoen and Jonsson (2003). Schoen (2006) explored the metastable model further, elaborating the model's features and noting the formal connection between the metastable birth trajectory and the normal curve. As a tool in applied demographic work, metastable models are particularly useful in analyzing steady fertility declines and for incorporating periods of transition between two regimes of fixed vital rates. 


\section{References}

Coale, A.J. (1972). The growth and structure of human populations. Princeton, NJ: Princeton University Press.

Coale, A.J. and Trussell, T.J. (1974). Model fertility schedules: Variations in the agestructure of childbearing in human populations. Population Index 40(2): 185-258. doi: $10.2307 / 2733910$.

Feichtinger, G. and Vogelsang, H. (1978). Pseudostabile Bevölkerungen: Populationsdynamik bei gleichmäßig sinkender Fertilität. Schriftreihe des Instituts für Demographie der Österreichischen Akademie der Wissenschaften, Vol 4. Vienna: Österreichische Akademie der Wissenschaften, Institut für Demographie.

Goldstein, J.R. (2002). Population momentum for gradual demographic transitions: An alternative approach. Demography 39(1): 65-73. doi:10.1353/dem.2002.0004.

Kim, Y.J. and Schoen, R. (1996). Populations with quadratic exponential growth. Mathematical Population Studies 6(1): 19-33. doi:10.1080/08898489609525419.

Li, N. and Tuljapurkar, S. (1999). Population momentum for gradual demographic transitions. Population Studies 53(2): 255-262. doi:10.1080/00324720308078.

Li, N. and Tuljapurkar, S. (2000). The solution of time-dependent population models. Mathematical Population Studies 7(4): 311-329. doi:10.1080/08898480009525464.

Lotka, A.J. (1998). Analytical theory of biological populations. Translated and with an Introduction by D.P. Smith and H. Rossert. New York: Plenum Press.

Preston, S.H., Heuveline, P., and Guillot, M. (2001). Demography: Measuring and modeling population processes. Oxford, UK: Blackwell.

Schoen, R. (1988). Modeling multigroup populations. New York: Plenum Press.

Schoen, R. (2006). Dynamic population models. Dordrecht: Springer.

Schoen, R. and Jonsson, S.H. (2003). Modeling momentum in gradual demographic transitions. Demography 40(4): 621-635. doi:10.1353/dem.2003.0040.

Schoen, R. and Kim, Y.J. (1994). Hyperstability. Paper presented at the Annual Meeting of the Population Association of America, Miami, FL, May 5-7, 1994.

Sykes, Z. (1973). Intrinsic age-specific birth rates: A new method of fertility analysis. [unpublished manuscript]. Baltimore, MD: Department of Population Dynamics, Johns Hopkins University.

Vogelsang, H. and Feichtinger, G. (1979). Exponentielle Niveau- und Musteränderung der Fertilität. Zeitschrift für Bevölkerungswissenschaft 1(1): 31-63. 